\title{
Management of acute epiglottitis in an infant with a family history of malignant hyperthermia
}

\author{
Michael Seltenrich, MD (D) James Capstick, MD • Clark Bartlett, MD
}

Received: 15 October 2015/Revised: 28 October 2015/Accepted: 18 November 2015/Published online: 7 January 2016

(c) Canadian Anesthesiologists' Society 2016

\section{To the Editor,}

We report our experience managing a previously healthy 16-mth-old female infant (weight, $11 \mathrm{~kg}$; with up to date immunizations) presenting to our regional hospital emergency department. She showed symptoms of fever, lethargy, and torticollis that had persisted for several hours but had no respiratory distress or drooling. A lateral neck $x$ ray showed a thumbprint sign (Figure), and the infant was diagnosed with acute epiglottitis. Of significance, the child's maternal grandfather had a positive muscle biopsy (caffeine-halothane contracture test) for malignant hyperthermia $(\mathrm{MH})$ after an $\mathrm{MH}$ crisis.

A decision was made to transfer the child to a pediatric hospital for definitive therapy but only after securing an airway. Accordingly, the infant was brought to the operating room in her mother's arms. As our main anesthetic goal was to maintain spontaneous ventilation while securing the airway, we considered using inhalational induction with a volatile agent as this was the technique with which we were most familiar. Nevertheless, the MH family history made this approach problematic. We briefly considered accompanying a volatile anesthetic induction with a prophylactic dantrolene treatment or simply monitoring for signs of MH after the airway was secured, but we recognized that the diagnosis of $\mathrm{MH}$ in a febrile, tachycardic, and possibly acidotic infant might be challenging.

M. Seltenrich, MD $(\varangle) \cdot$ J. Capstick, MD

Department of Anesthesia, Nanaimo Regional General Hospital,

Nanaimo, BC, Canada

e-mail: Michael.Seltenrich@viha.ca

C. Bartlett, MD

Department of Surgery, Nanaimo Regional General Hospital,

Nanaimo, BC, Canada
We considered a total intravenous anesthesia (TIVA) technique as an alternative. In our institution, this usually involves bolus doses of propofol, narcotics, and muscle relaxants that render the patient apneic during airway management. Although spontaneous ventilation during a TIVA induction with propofol, remifentanil, and sometimes dexmedetomidine is used routinely in some pediatric hospitals, ${ }^{1,2}$ we had limited experience with this approach. Nevertheless, we ultimately decided that this was the better option.

Using the methods of Malherbe et al., ${ }^{1}$ we placed an intravenous catheter (after applying topical lidocaine to the infant's skin) while the child was being comforted upright in her mother's arms. Ceftriaxone $1 \mathrm{gm}$ was given intravenously. After placing a pulse oximeter and applying blow-by oxygen, we began a propofol infusion at $300 \mu \mathrm{g} \cdot \mathrm{kg}^{-1} \cdot \mathrm{min}^{-1}$ and a separate remifentanil infusion at $0.05 \mu \mathrm{g} \cdot \mathrm{kg}^{-1} \cdot \mathrm{min}^{-1}$. As the patient became drowsy, we transferred her to the operating table in a supine position with her airway supported with a face mask and then placed the remaining standard monitors. ${ }^{3}$ Only a moderate amount of chin lift was required, and continuous positive airway pressure was not necessary during induction. The remifentanil infusion was gradually increased (maximum rate $0.3 \mu \mathrm{g} \cdot \mathrm{kg}^{-1} \cdot \mathrm{min}^{-1}$ ), and after $20 \mathrm{~min}$, the infant's respiratory rate had decreased from 40 to 20 breaths $\cdot \mathrm{min}^{-1}$. Video laryngoscopy (GlideScope ${ }^{\circledR}$; Verathon Inc., Bothell, WA, USA) showed a laryngeal orifice that was not well visualized along with arytenoids that were swollen and covered with exudate; however, tracheal intubation (\#4 cuffed endotracheal tube) was accomplished uneventfully. There were no episodes of apnea, breath holding, coughing, or arterial desaturation. The infant was transferred to the tertiary care pediatric hospital where she made an 


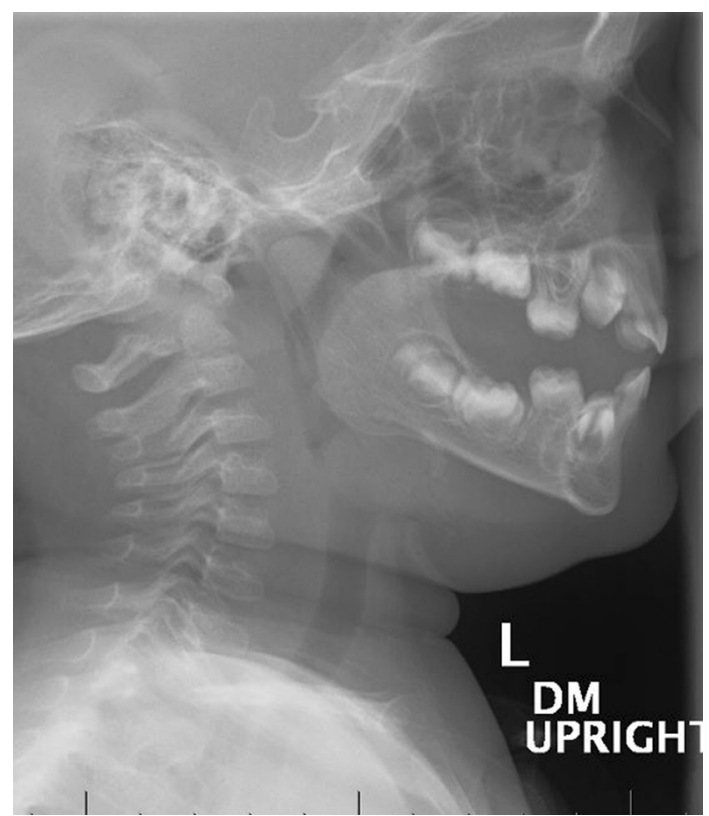

Figure A lateral neck $x$-ray showing a classic thumbprint sign consistent with acute epiglottitis

uneventful recovery. A culture for Haemophilus influenzae was not obtained.

There are a number of scenarios where spontaneous ventilation during induction is desirable in children, including acute airway infections, airway tumours, foreign bodies, and inhalational injuries. In patients with a family history of $\mathrm{MH}$, a TIVA induction with spontaneous breathing may be the best option if awake intubation is not possible. ${ }^{4}$ In our view, any anesthesiologists who may need to deal with pediatric airway emergencies should familiarize themselves with this technique.

Conflict of interest The authors declare no conflict of interest.

\section{References}

1. Malherbe S, Whyte S, Permendra S, Amari E, King A, Ansermino $J M$. Total intravenous anesthesia and spontaneous respiration for airway endoscopy in children-a prospective evaluation. Pediatr Anesth 2010; 20: 434-8.

2. Chen $K Z, Y e M, H u C B$, Shen $X$. Dexmedetomidine vs remifentanil intravenous anaesthesia and spontaneous ventilation for airway foreign body removal in children. Br J Anaesth 2014; 112: 892-7.

3. Merchant $R$, Chartrand D, Dain $S$, et al. Guidelines to the practice of anesthesia-revised edition 2015. Can J Anesth 2015; 62: 5479.

4. Allen GC, Byford LJ, Shamji FM. Anterior mediastinal mass in a patient susceptible to malignant hyperthermia. Can J Anaesth 1993; 40: 46-9. 